\title{
Seguimiento de Egresados en Tres Programas de Maestría en una Escuela del Instituto Politécnico Nacional en México
}

\author{
Claudia A. Hernández*, María E. Tavera y Martha Jiménez \\ Instituto Politécnico Nacional, Unidad Profesional Interdisciplinaria de Ingeniería y Ciencias \\ Sociales y Administrativas (UPIICSA), Sección de Estudios de Posgrado e Investigación, \\ Área Maestría en Administración, México, Distrito Federal (e-mail: al9505@gmail.com, \\ mtavera@ipn.mx, majimenez@ipn.mx).
}

*Autor a quien debe ser dirigida la correspondencia.

Recibido Oct. 19, 2011; Aceptado Dic. 13, 2011; Versión final recibida Dic. 15, 2012

\section{Resumen}

Se presentan los resultados de un estudio para conocer la mejora laboral y de ingresos que obtienen actualmente los graduados de las maestrías. El estudio consideró tres programas de maestría de cinco generaciones en una Escuela de Educación Superior perteneciente al Instituto Politécnico Nacional en la Ciudad de México. El método que se trabajó fue cuantitativo de tipo descriptivo y transversal. Se utilizó un cuestionario, el cual fue diseñado tomando en cuenta algunos factores del esquema básico de estudios de egresados propuesto por la Asociación Nacional de Universidades e Instituciones de Educación Superior. En conclusión, los egresados de las tres maestrías consiguen mejorar su situación laboral después de terminar sus estudios, Los ingresos de los ex-alumnos tienen una mejoría que varía según el programa que hayan cursado.

\section{Graduates Follow-Up in Three Master Degree Programs at the National Polytechnic Institute in Mexico}

\begin{abstract}
The results of a study to know the improvement in labor and earnings in their jobs of persons graduated from masters programs are presented. The study considered three master programs of five generations of the School of Higher Education belonging to the National Polytechnic Institute in Mexico City. The method used was quantitative, descriptive and transversal. A questionnaire designed to take into account basic scheme factors of graduates proposed by the Mexican National Association of Universities and Higher Education Institutions was used. In conclusion, the graduates from these three master degrees improved their labor situation after concluding their studies, while the increase in salaries varies depending on the program they followed.
\end{abstract}

Keywords: graduates follow-up, graduate studies, labor situation, monthly earning 


\section{INTRODUCCIÓN}

La educación de posgrado en un conjunto de procesos de enseñanza-aprendizaje dirigidos a garantizar la preparación de los graduados universitarios para complementar, actualizar y profundizar los conocimientos y habilidades que poseen, vinculados directamente al ejercicio profesional, los avances científicos-técnicos y las necesidades de las entidades en las que laboran (Manzo, Rivera y Rodríguez, 2006). Los objetivos principales de los programas de posgrado a nivel de maestría es proporcionarles a los alumnos una formación amplia y sólida en la disciplina, con la finalidad de iniciarlos en el ámbito de la investigación, para el ejercicio de la docencia y la especialización en el campo profesional (Sánchez y Arredondo, 2001).

En México los estudios de posgrado se dividen en tres: 1) especialización, maestría y doctorado. El tiempo de duración es de doce, veinticuatro y treinta y seis meses respectivamente. Las escuelas públicas a nivel nacional que imparten programas de posgrado son 836, además se cuenta con un sistema privado el cual se integra de 817 planteles. La matricula que se atendió en el 2008 fue de 185,486 alumnos, de los cuales el $21.45 \%$ se encontraban estudiando una especialidad, el $68.57 \%$ una maestría y tan sólo el 9.98\% un doctorado. La matrícula de posgrado fue atendida en un $51.20 \%$ por el sistema público y en un $48.8 \%$ por el sector privado (SEP, 2009).

Los egresados son el producto final que se obtiene después del transitar por los programas de posgrado. El camino que deciden tomar los egresados, su crecimiento y desempeño profesional son factores que deben de ser estudiados por las escuelas, para reflexionar sobre los resultados obtenidos de la educación alcanzada. Los estudios de egresados coadyuvan a la construcción de un diagnóstico que sirve para estudiar las tendencias de ocupación, sueldo, tiempo que se tardan en colocarse en el mercado laboral, satisfacción con la formación recibida y la aplicación de sus conocimientos adquiridos en su actual empleo. La Asociación Nacional de Universidades e Instituciones de Educación Superior (ANUIES, 1998) menciona que los estudios de egresados que se han realizado son muy heterogéneos en cuanto a su metodología. Además, las limitaciones que se pueden presentar en este tipo de estudios son: el alto costo lo que los ubica con una baja prioridad dentro del presupuesto institucional; el alto grado de dificultad cuando no se cuenta con una base de datos para localizar ágilmente a los egresados y la carencia de expertos en este tipo de investigaciones. Tales razones hacen que este tipo de estudios no se realicen de manera frecuente.

Algunos estudios de egresados en programas de posgrado muestran la situación laboral actual de los ex alumnos lo que permite saber la relación entre los estudios alcanzados y la actividad que desempeñan. El status académico, lo que ayuda a identificar si son egresados que tienen grado o no. Alumnos que trabajaban durante sus estudios y los impactos que pueden tener las becas disfrutadas con relación a los objetivos alcanzados (Ramírez, Reyna, García, Ortiz y Valdez, 2011; Figueroa, Bernal y Andrade, 2010; Luchilo, 2009; Martínez, Moreno, Hernández, Miguel y Martínez, 2005). Se encontraron tres investigaciones de seguimiento de egresados que fueron realizadas en la Unidad Profesional Interdisciplinaria de Ingeniería y Ciencias Sociales y Administrativas (UPIICSA), la primera, explica los obstáculos y beneficios de trabajar un esquema para estudiar a los egresados (Rodríguez y Fernández, 1986), la segunda, analiza la calidad del posgrado en administración desde la óptica del egresado, aquí se estudian factores como; 1) la situación laboral antes y después del posgrado, 2) logro de expectativas y 3) materias más útiles para el egresado para su desempeño en el trabajo (Fernández, 1993) y en la tercera se recopilan opiniones de los ejecutivos con relación a la calidad del desempeño de los egresados (Fernández, 1992). Las investigaciones antes mencionadas datan de hace dieciocho años, lo que significa que durante todo ese tiempo no se han vuelto a tener contacto con los egresados. Es de suma valía que la escuela diseñe una planeación adecuada que contenga tiempos programados para comenzar con el seguimiento de egresados. En la presente investigación, se entiende por egresado a: 1) la persona que ha concluido el $100 \%$ de los créditos señalados en el plan de estudios, pero no tiene el grado de maestro y 2) el estudiante que ha terminado el $100 \%$ de créditos y que cuenta con el grado de maestría. 
Las investigaciones revisadas ofrecen resultados que orillan a la reflexión generalizada sobre el comportamiento a través del tiempo de los programas de posgrado analizados, pero no evalúan factores relacionados con el avance o retroceso económico de los egresados durante y después del posgrado.

El Consejo Nacional de Ciencia y Tecnología (CONACyT) es la institución que se encarga de elaborar políticas encaminadas a fortalecer el desarrollo científico y tecnológico de México. Además, destina recursos para los programas de posgrado que consiguen acreditar su calidad mediante la evaluación de diecinueve lineamientos. Las instituciones que garantizan que los posgrados que se imparten cuentan con un núcleo básico de académicos, altas tasas de graduación, infraestructura necesaria, alta productividad científica y tecnológica y el seguimiento de egresados, entre otros, ingresan al Programa Nacional de Posgrados de Calidad (PNPC).

El Instituto Politécnico Nacional realiza su seguimiento de egresados a través del Sistema Institucional de Seguimiento y Actualización de Egresados (SISAE), el sistema sólo genera resultados con relación a los niveles de enseñanza media y superior, no contempla a los ex alumnos de especialidad, maestría y doctorado.

La Unidad Profesional Interdisciplinaria de Ingeniería y Ciencias Sociales y Administrativas (UPIICSA) del Instituto Politécnico Nacional (IPN), cuenta con la Sección de Estudios de Posgrado e Investigación (SEPI) en la cual operan cuatro programas de posgrado en el nivel de maestría, dos tienen el registro en el Programa Nacional de Posgrados de Calidad (PNPC) del Consejo Nacional de Ciencia y Tecnología (CONACyT). La exigencia de las evaluaciones de los criterios que realiza CONACyT, la re-estructuración de los programas de estudios en el 2007 y la falta de investigaciones que estudien a los egresados de posgrado en la UPIICSA, llevó a focalizar el interés en cinco generaciones de ex alumnos del 2004 al 2008 de tres programas de maestría.

El presente estudio de investigación, busca obtener un diagnóstico de egresados que permita conocer la posible mejora laboral y salarial de los egresados de tres programas de posgrado en la Unidad Profesional Interdisciplinaria de Ingeniería y Ciencias Sociales y Administrativas (UPIICSA). Se reconoce la importancia del seguimiento de egresados como una herramienta básica para el diagnóstico, la mejora de los planes de estudio y la definición de políticas de desarrollo institucional ante las nuevas necesidades sociales (Valentí y Varela, 2004; Martínez, et al., 2005). Además, son mecanismos poderosos de diagnóstico de la realidad con el potencial de inducir en las instituciones la reflexión a fondo sobre sus fines y sus valores. Los resultados de estos estudios pueden asimismo, aportar elementos para redefinir el proyecto de desarrollo de aquellas instituciones que se mantienen alerta ante las nuevas necesidades sociales, permitiéndoles reconocer y asumir las nuevas formas de práctica profesional que se requieren para sustentar un proceso social menos inequitativo y dependiente (Fresán, 1998).

\section{MATERIALES Y MÉTODOS}

El método que se ocupó fue cuantitativo de tipo transversal descriptivo. Los diseños de investigación transversal recolectan datos en un sólo momento, en un tiempo único. Su propósito es describir variables y analizar su incidencia e interrelación en un momento dado (Hernández. Fernández y Baptista, 2006). La obtención de datos se realizó a través de la construcción de un cuestionario, el cual se compone de 23 reactivos distribuidos en cinco bloques: 1) datos generales, 2) situación laboral y de ingresos durante y después de la maestría, 3) status académico con o sin grado, 4) aplicación de conocimientos en el campo laboral, 5) calificación al programa de maestría cursado. En la elaboración del cuestionario se consideró algunos factores del Esquema Básico de Estudio de Egresados de la ANUIES (1998) y variables enunciadas en la metodología para realizar estudios de egresados en el IPN. La validación del cuestionario fue a través de la revisión por un experto y pionero en seguimiento de egresados en la UPIICSA.

Los cuestionarios fueron enviados por correo electrónico y el tiempo de respuesta por parte de los alumnos fue de siete días. Se consideraron las generaciones del 2004 al 2008 debido a que han sido los grupos de alumnos que permitieron cimentar las bases en la preparación del terreno para la evaluación de los indicadores que nos permitieron ingresar al Programa de Posgrados de 
Calidad (PNPC) del Consejo Nacional de Ciencia y Tecnología. La recolección de los datos fue realizada en los meses de febrero a junio del año 2011. La información fue capturada y analizada en Excel.

El muestreo se hizo según los criterios de Fox y López (1981). En primer lugar se obtuvo el universo de los egresados de las tres maestrías de los años 2004 al 2008, teniendo un total de 320 alumnos. En segundo lugar, se fijaron dos criterios para seleccionar a los alumnos que pudiesen contestar el cuestionario: 1) egresados con el $100 \%$ de créditos concluidos y 2) alumnos que tuvieran su grado de maestros con un total de 196. Se enviaron los 196 cuestionario vía correo electrónico invitando a los egresados a participar en el estudio. La muestra productora de datos fue de 67 personas que accedieron a responder la petición solicitada. 34 de administración, 24 de ingeniería industrial y 7 de informática.

\section{RESULTADOS}

De la población encuestada se obtuvo que el $64.20 \%$ son hombres y el $35.80 \%$ mujeres. El promedio de edad de los egresados es de 33.8 años con una desviación estándar de 5.8 años. El programa de maestría que obtuvo mayor participación de los egresados fue el de administración con un 53.7\%, en segundo lugar el de ingeniería industrial con el 35.8\% y por último el $10.40 \%$ de informática. La ocupación actual de los egresados se encuentra en la empresa privada, con un $50.7 \%$, el $35.8 \%$ son empleados del gobierno, el $4.5 \%$ es consultor independiente, el $7.5 \%$ está desempleado y el $1.5 \%$ son mujeres que no desean trabajar por el momento.

El seguimiento de egresados de la UPIICSA-IPN muestra que el $52.2 \%$ de los encuestados ya obtuvo el grado de maestría y el $47.8 \%$ no lo tiene. El $70 \%$ señala que fue alumno de tiempo parcial y el $30 \%$ de tiempo completo. Con relación a los apoyos económicos que son las becas el $18 \%$ dice que sí tenía beca y el $82 \%$ señala que no.

La situación laboral de los egresados durante sus estudios de maestría refleja que el $77 \%$ contaba con empleo y el $22 \%$ no tenía trabajo. De los que laboraban, el $51 \%$ refiere que trabajaba para una empresa privada, el $16 \%$ en el gobierno, el $8 \%$ en el sector de los servicios y sólo el $3 \%$ se empleaban por su cuenta. Los ingresos promedio que los alumnos tenían en la etapa de estudiantes se encuentran en una media de $\$ 15,692$ pesos mexicanos con una desviación estándar de \$6,596.

El 77\% de los egresados tenían trabajo antes de entrar y también al salir. Pero este trabajo puede ser distinto no necesariamente el mismo. El tiempo promedio que se tardaron en encontrar trabajo los egresados fue de 1 a 3 meses, lo que indica una rápida incorporación a la actividad productiva. El escenario laboral de los egresados es positivo, el 91\% tiene empleo y sólo el 9\% se encuentra desempleado. El promedio de ingresos actual está en una media de $\$ 19,733$ pesos con una desviación de \$6,910.

El estudio arroja que el $77.60 \%$ de los encuestados está de acuerdo con la creencia de que los estudios de maestría ayudan a mejorar la situación laboral y su inmersión al campo productivo, el $22.40 \%$ no está de acuerdo. El número de encuestados que dice aplicar los conocimientos adquiridos en el posgrado en su trabajo cuadriplica a los que no. Un dato importante es que el $76 \%$ de los egresados se encuentran interesado en cursar algún programa de estudios a nivel de doctorado.

El $86.60 \%$ de los egresados dice que el programa de maestría que cursaron logró satisfacer sus expectativas de conocimientos, el $13.40 \%$ refiere que no. La calificación que le dan a los posgrados es positivo, el 52.20\% le otorga una calificación de "bien" (entre 8 y 9), el 35.80\% dice que fue excelente (10) y el $11.90 \%$ refiere que fue "regular" (entre 7 y 8 ).

\section{RESULTADOS COMPARATIVOS DE LAS TRES MAESTRÍAS}

Los egresados que participaron en el estudio se encuentran en una edad madura y productiva, la maestría en ingeniería industrial apuntala con una media aritmética de 35 años, en segundo lugar 
la maestría en administración con un promedio de 33.6 años y el grupo que se considera como más joven es la maestría en informática con una media de 33 años.

Los egresados del programa de administración y de ingeniería industrial se mueven en una horquilla del 55.6 al 54.2 en obtención del grado académico, prácticamente doblando a la maestría en informática (Ver figura 1).

Cuenta con grado $₫$ Sin grado

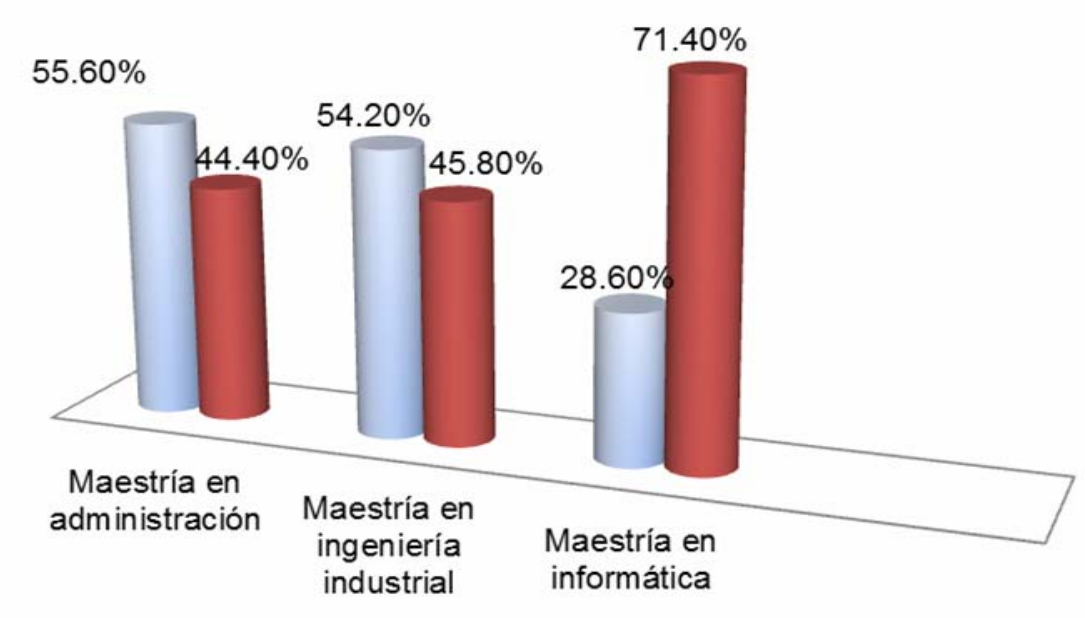

Fig. 1: Comparativo de los egresados con grado y sin grado académico

El análisis arrojó que durante la estancia en la maestría, el posgrado en informática tiene el mayor porcentaje de alumnos que tenían empleo con un $85.7 \%$, no tan lejana está la maestría en administración con el $83.3 \%$ y por último el $66.7 \%$ de los egresados en ingeniería industrial señalaron que trabajan.

A los egresados se les cuestionó en que sector se emplearon durante sus estudios de posgrado, se encontró que en promedio la mayoría de ellos estaban en la empresa privada. La maestría en administración muestra el mayor porcentaje de trabajadores en el sector privado con el 61.1\%, enseguida están los egresados en ingeniería industrial con el 41.7\%. Los informáticos son los que tienen mayor presencia en el sector gobierno con un 42.9\% (Ver figura 2).

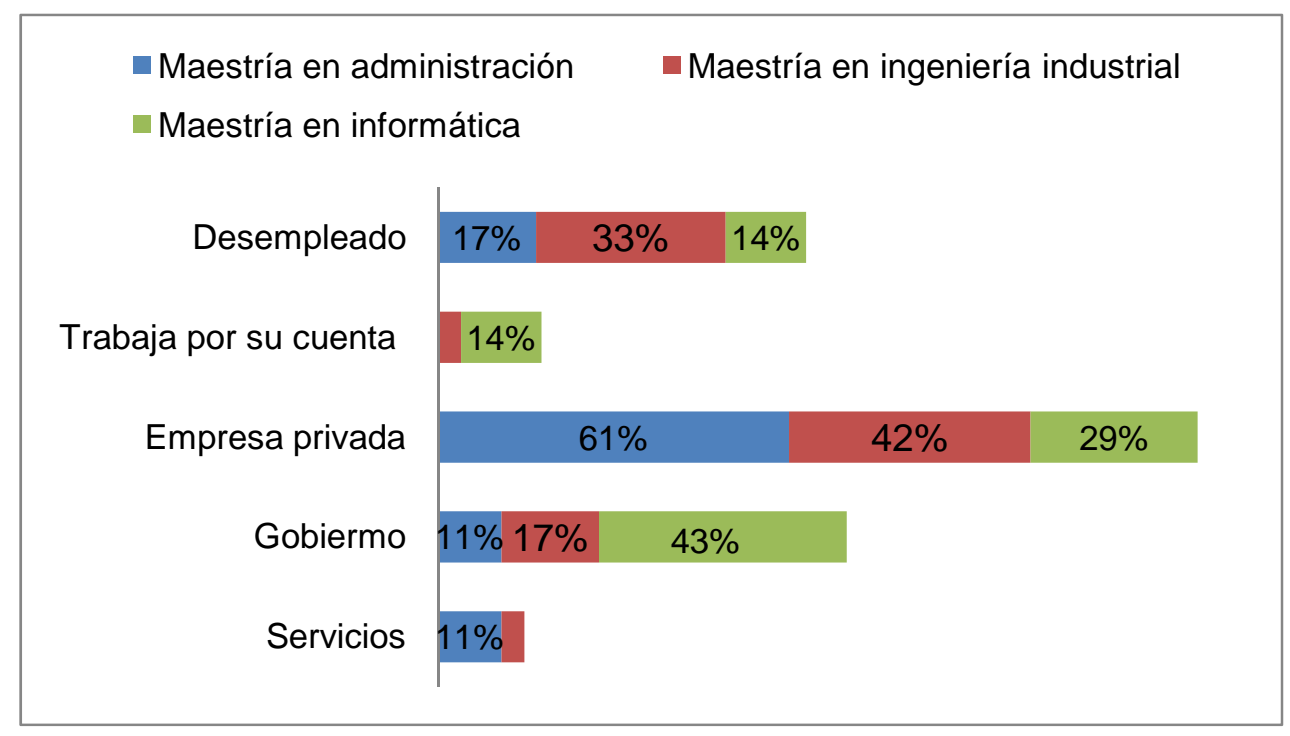

Fig. 2: Sector en que se empleaban los alumnos durante sus estudios de maestría 
La investigación halló que un porcentaje significativo de alumnos en las tres maestrías no buscaron trabajo, en general ya lo tenían y lo conservan. El grupo de los informáticos con el $85.7 \%$ no tuvieron la necesidad de buscar otro empleo, le siguen los administradores con el 80.6\%. Se observa movilidad laboral en el programa de ingeniería industrial, el 37.5\% comenzaron a buscar un espacio laboral y el $62.5 \%$ se quedó en su trabajo (Ver figura 3 ).

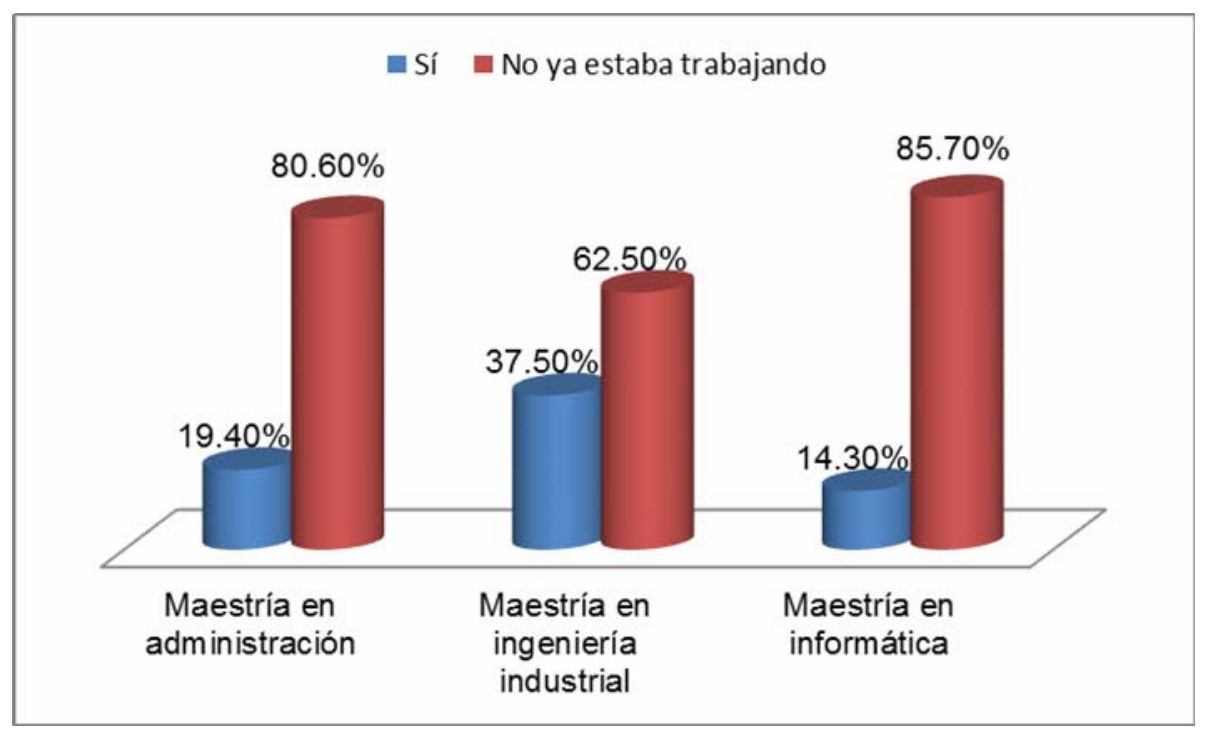

Fig. 3: Egresados que buscaron empleo al finalizar los estudios

El estudio encontró que el tiempo máximo que se tardan los egresados de la maestría en administración e informática en encontrar trabajo es de 1 a 3 meses. El posgrado en informática tarda entre 7 a 9 meses. Lo que significa, que los egresados de los posgrados de los tres programas tienen una aceptable demanda e inserción en el mercado laboral.

La investigación encuentra que el porcentaje de ocupación en el empleo es alta. La maestría en informática es la que encabeza con el $100 \%$, el programa de administración tiene el $91.7 \%$ de sus egresados ocupados y la maestría en ingeniería industrial refleja que el $87.5 \%$ de sus ex estudiantes están empleados. Se encuentra una mejoría con la situación laboral con relación al antes y el después de haber cursado la maestría. Los egresados de la maestría en ingeniería industrial son los que obtienen una mejoría considerable.

El mayor porcentaje de los egresados en los tres programas de posgrado siguen prefiriendo la empresa privada seguido por el trabajo en el gobierno. Los administradores por la naturaleza de su competencia en el ámbito empresarial encabezan la lista con un $58.3 \%$ de egresados que se colocan en el sector privado, los ingenieros se emplean en la industria con el 45.8\%. Los informáticos tienen mayor presencia en el gobierno con el $71.4 \%$.

Los participantes en el estudio refieren en su mayoría que los conocimientos adquiridos durante la maestría los aplican en su trabajo. Los encuestados del programa de maestría de informática son los que mejor ocupan los conocimientos con el $85.7 \%$, en segundo lugar está la maestría en administración con un $77.8 \%$ y en tercer lugar los egresados de ingeniería industrial con el $70.8 \%$ (Ver tabla 9).

Los egresados en los tres grupos perciben que el estudiar un posgrado es un mecanismo que apoya a mejorar su situación laboral y que incluso coadyuva a encontrar trabajo. La satisfacción de los egresados es un indicador de suma valía para cualquier programa educativo en operación. Los datos demuestran un escenario optimista para los tres programas de posgrado estudiados, en su mayoría los egresados refieren que el plan de estudios logró satisfacer sus expectativas de conocimientos, lo que indica que se tienen calidad en la diversidad de actividades involucradas en la enseñanza y formación de recursos humanos de alto nivel (Ver tabla 11). La calificación que los 
egresados a los programas de maestría que cursaron en su mayoría se recargan en un nivel de "buena" entre 8 y 9. Este resultado refuerza la satisfacción favorable que perciben los encuestados (Ver tabla 12).

Se efectúo un análisis de los sueldos durante y después del posgrado. Se quiere saber si la terminación de los estudios de posgrado ayuda a que los sueldos de los egresados se incrementen (Ver tabla 1).

Tabla 1. Comparativo de los sueldos de los egresados durante y después de la maestría

\begin{tabular}{|c|c|c|c|c|c|c|}
\hline \multirow[b]{2}{*}{$\begin{array}{l}\text { Situación } \\
\text { sueldo }\end{array}$} & \multicolumn{2}{|c|}{ Maestría en administración } & \multicolumn{2}{|c|}{$\begin{array}{c}\text { Maestría en ingeniería } \\
\text { industrial }\end{array}$} & \multicolumn{2}{|c|}{ Maestría en informática } \\
\hline & Durante la maestría & Después & $\begin{array}{l}\text { Durante la } \\
\text { maestría }\end{array}$ & Después & $\begin{array}{l}\text { Durante la } \\
\text { maestría }\end{array}$ & Después \\
\hline Media & $\$ 16,064.51$ & $\$ 21,470.58$ & $\$ 10,357.14$ & $\$ 16,473.68$ & $\$ 15,714.28$ & $\$ 20,142.85$ \\
\hline $\begin{array}{l}\text { Desviación } \\
\text { estándar }\end{array}$ & $\$ 7,509.70$ & $\$ 10,652.17$ & $\$ 5,245$ & $\$ 8,559.39$ & $\$ 5,089$ & $\$ 8,234.65$ \\
\hline
\end{tabular}

Tabla 2. Comparativo de sueldos en pesos mexicanos durante y después de la maestría de acuerdo con la generación de egreso

\begin{tabular}{|l|r|r|r|r|r|r|}
\hline \multirow{2}{*}{$\begin{array}{c}\text { Generación de } \\
\text { egreso }\end{array}$} & \multicolumn{2}{|c|}{ Maestría en administración } & \multicolumn{2}{c|}{$\begin{array}{c}\text { Maestría en ingeniería } \\
\text { industrial }\end{array}$} & \multicolumn{2}{c|}{ Maestría en informática } \\
\cline { 2 - 7 } & Durante la maestría & $\begin{array}{l}\text { Actual (año } \\
2011)\end{array}$ & $\begin{array}{l}\text { Durante la } \\
\text { maestría }\end{array}$ & $\begin{array}{l}\text { Actual (año } \\
2011)\end{array}$ & $\begin{array}{l}\text { Durante la } \\
\text { maestría }\end{array}$ & $\begin{array}{l}\text { Actual (año } \\
2011)\end{array}$ \\
\hline $2004-2006$ & $\$ 12,285.71$ & $\$ 23,000$ & $\$ 14,000$ & $\$ 16,600$ & $\$ 18,000$ & $\$ 27,000$ \\
\hline $2005-2007$ & $\$ 15,000$ & $\$ 21,400$ & $\$ 16,000$ & $\$ 15,000$ & $\$ 22,000$ & $\$ 31,000$ \\
\hline $2006-2008$ & $\$ 18,571.42$ & $\$ 21,666$ & $\$ 14,000$ & $\$ 11,000$ & $\$ 15,333$ & $\$ 17,666$ \\
\hline $2007-2009$ & $\$ 18,571.42$ & $\$ 24,142.85$ & $\$ 18,000$ & $\$ 23,000$ & 0 & 0 \\
\hline $2008-2010$ & $\$ 15,333.33$ & $\$ 17,500$ & $\$ 14,666.66$ & $\$ 18,000$ & $\$ 12,000$ & $\$ 15,000$ \\
\hline
\end{tabular}

Según la tabla 2 se observa que en la mayoría de las generaciones hay una mejoría positiva, sin embargo, se reconoce la pérdida del valor del dinero con relación al tiempo, por lo anterior, se efectúo un cálculo con respecto al número de salarios mínimos que percibían y perciben los egresados. De acuerdo con la Secretaría de Hacienda y Crédito Público el salario mínimo vigente en la zona "A" es el siguiente: 1) 2004 con \$45.24 pesos, 2) 2005 con \$46.80, 3) 2006 con 48.67, 4) 2007 con 50.57 , 5) 2008 con 52.59 pesos y 6) 2011 con 59.82 pesos. Se debe de señalar que no hubo egresados de la generación 2007-2009 en la maestría en informática que quisieran participar en el estudio.

Los resultados que se obtuvieron demuestran que la maestría en administración en las generaciones del 2004, 2005, 2006, 2007 y 2008 incrementan en pequeña proporción el número de los salarios mínimos que obtienen en el 2011. La maestría en administración presenta subidas en el salario mínimo en las generaciones 2004, 2005 y 2007. En el caso de informática, sólo las generaciones del 2004, 2005 y 2008 se logran ligeros aumentos. La maestría en ingeniería industrial es una de la más golpeadas en las generaciones de 2004, 2005 y 2006 no se observa ninguna mejoría, tan sólo se tienen precarios cambios en las generaciones del 2007 y 2008. 


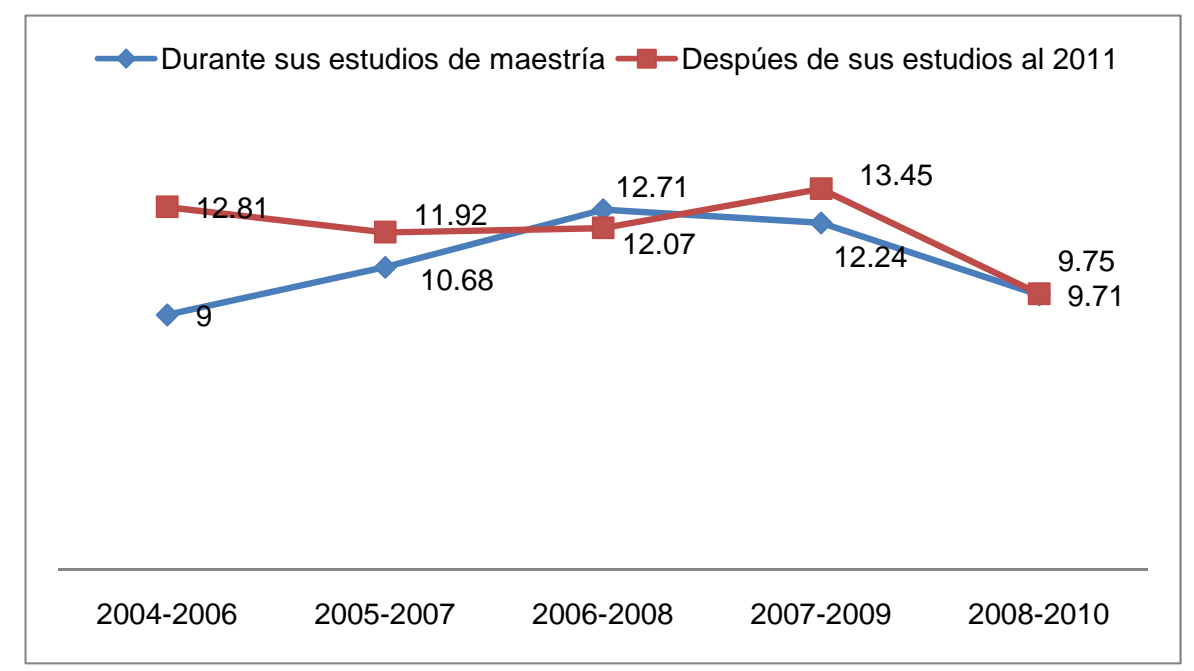

Fig. 4: Comparativo del número de salarios mínimos diarios que perciben los egresados de la maestría en administración durante y después de los estudios de posgrado

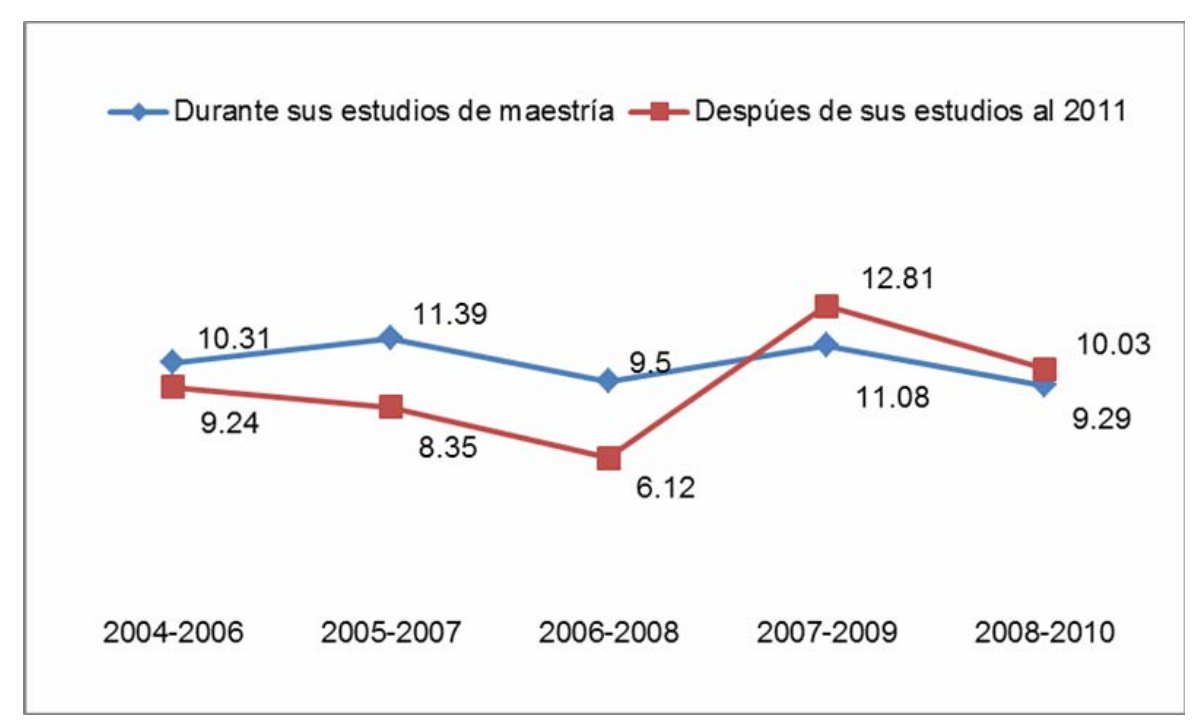

Fig. 5: Comparativo del número de salarios mínimos diarios que perciben los egresados de la maestría en ingeniería industrial durante y después de los estudios de posgrado

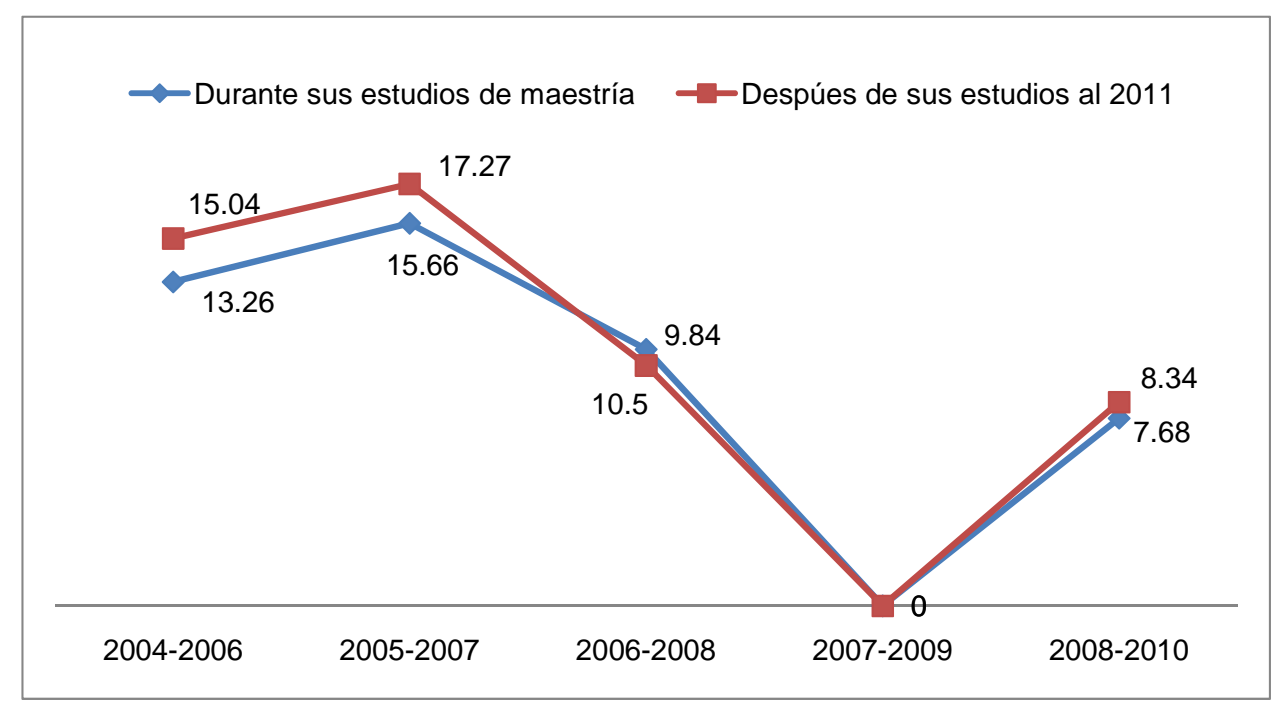

Fig. 6: Comparativo del número de salarios mínimos diarios que perciben los egresados de la maestría en informática durante y después de los estudios de posgrado 


\section{DISCUSIÓN}

El seguimiento de egresados en una poderosa herramienta que permite visualizar el escenario que viven los ex alumnos en su vida laboral y la forma en que esta impacta directamente en su bienestar económico. El esfuerzo y dedicación de concluir un posgrado debe de representar la apertura de mejores oportunidades que conlleven al crecimiento laboral. Cuando el egresado progresa la institución también avanza. La investigación mostró los adelantos en la situación laborales, entonces se observa la relación entre educación y empleo mencionada por la teoría del capital humano. La teoría enfatiza que las variaciones entre salarios están en función del nivel educativo formativo alcanzado. La idea básica es considerar a la educación y la formación como inversiones que realizan individuos racionales, con el fin de incrementar su eficiencia productiva y sus ingresos (Destinobles, 2006).

El estudio hace evidente que sí hay incremento en los sueldos de los egresados, se tienen generaciones en donde los ex alumnos registran una mayor cantidad de salarios mínimos al día. A los egresados de las maestrías les favorece de forma positiva el hecho de haber terminado sus estudios. De acuerdo con la Encuesta Nacional de Ocupación y Empleo (ENOE) en el segundo trimestre los profesionistas con nivel de licenciatura dedicados a la administración ganan al mes $\$ 9,933$, es decir 5.53 salarios mínimos, los ingenieros industriales $\$ 10,933$ que representan 6.09 salarios mínimos y los informáticos alcanzan un sueldo de $\$ 9,080$ que son 5.05 salarios mínimos. Efectuando el análisis del número de salarios mínimos que ganan en promedio y al día los trabajadores con licenciatura se tiene que los egresados de la maestría en administración se encuentran ganando el doble, los ex alumnos de la maestría en ingeniería industrial están obteniendo dos salarios mínimos más al día y para la maestría en informática se observa que perciben dos veces más que un profesionista con menor nivel de escolaridad.

La investigación tiene como limitante que el cuestionario aplicado a los ex alumnos no pregunta su posicionamiento dentro del organigrama de la empresa en la que laboran (estratégico, táctico u operativo) y tampoco considera la complejidad de actividades, ni la opinión de los empleadores y la antigüedad laboral.

El ingreso y permanencia de los egresados en el trabajo en un fuerte indicador que muestra las posibles preferencias de los empleadores. Se encontró que un $91 \%$ los egresados de las maestrías están ocupados por los mercados de trabajo, al respecto Ávila y Aguirre (2005) mencionan que cuanto mayor sea el índice de ocupación de los egresados mayor será la calidad de la enseñanza, pero se requiere una dinámica demandante y favorable del mercado laboral.

El tiempo de permanencia que tienen los individuos en un empleo ayuda al incremento de conocimientos y experiencias de un área específica de trabajo, al respecto Fernández, Reyes, Dávila y Torres (2006) encontraron que a medida que los egresados permanecen más tiempo en un trabajo, logran cambios favorables como aspectos tales como el salario, grado de coincidencia del trabajo con sus formación profesional y el tipo de contratación. Se coincide con esta investigación, ya que en nuestro estudio se halló que el $77 \%$ de los egresados conservan su empleo con el cual comenzaron la maestría, lo que indica que la permanencia en el trabajo coadyuva a su desarrollo profesional.

Los posgrados que se encuentran inscritos al Programa Nacional de Posgrados de Calidad (PNPC) del CONACYT deben de efectuar evaluaciones constantes para analizar la pertinencia del plan de estudios y la proyección de los ex alumnos en el ámbito laboral. La satisfacción que el egresado percibe con respecto a la formación que recibió durante su tránsito por las aulas de la institución es un indicador que aporta información que conlleva a la reflexión del cuerpo académico encargado de transmitir conocimientos. Al respecto, Fresán (1998), señala que los estudios de egresados son poderosos mecanismos que contribuyen indirectamente a evidenciar la calidad de la planta académica de las instituciones educativas, actualidad de sus planes y programas de estudio y de la idoneidad de sus estrategias pedagógicas. La reflexión que se tiene después de haber obtenido un $86.60 \%$ de alumnos satisfechos con sus expectativas de conocimientos, está dirigida a fortalecer y dar a conocer este tipo de evaluaciones a los profesores 
de la sección de posgrado, para que den cuenta de la significancia de su labor cotidiana que realizan en las aulas.

Las implicaciones del presente trabajo son: a) difundir la importancia de este tipo de investigaciones para lograr la participación activa de los alumnos y así lograr el acercamiento con los empleadores y b) la adecuada divulgación de los resultados para fomentar la reflexión de los agentes involucrados en la operación de los programas de posgrado en la UPIICSA.

\section{CONCLUSIONES}

Del estudio llevado a cabo se obtienen las siguientes conclusiones:

1. Se logró conocer los avances laborales y económicos de los alumnos de las tres maestrías analizadas. Con la información obtenida, se gana terreno para fortalecer los programas de estudio y la práctica docente. La investigación comprueba que los alumnos que consiguen terminar sus estudios de posgrado tienen repercusiones positivas en su vida laboral.

2. El seguimiento permite reconocer los problemas que se tienen con respecto al porcentaje elevado de los egresados que no han obtenido el grado académico, por lo anterior, es posible pensar en alternativas que sean factibles de implementar para apoyar a los ex alumnos en titularse.

3. Es imprescindible trabajar en los ítems que consideren datos sobre la ubicación jerárquica y funciones de los egresados. Además, de trabajar en la elaboración de un cuestionario enfocado a los empleadores.

4. El estudio de egresados necesita una adecuada difusión entre los alumnos para lograr su participación.

5. Para continuar con el seguimiento de los egresados, es importante tener una base de datos actualizada que permita tener contacto con ellos y con sus empleadores.

\section{REFERENCIAS}

Asociación Nacional de Universidades e Instituciones de Educación Superior. Esquema Básico para estudios de egresados, $1^{a}$ edición, Colección Bibliotecas de la Educación Superior (ANUIES), (31-58), México, (1998).

Ávila, M. y Aguirre, C. El seguimiento de egresados como indicador de la calidad docente, Revista Electrónica Interuniversitaria de formación del profesorado, ISSN: 1575-0965 (en línea), 8(3) mayo, (2005), http://redalyc.uaemex.mx/redalyc/src/inicio/ArtPdfRed.jsp?iCve=217017156006. Acceso 24 de mayo (2011).

Destinobles, A. El capital humano en las teorías del crecimiento económico, $1^{\mathrm{a}}$ edición, (21-23), eumed.net, Málaga, España (2006).

Fernández, J., Reyes, K, Dávila, C. y Torres, O. Maestros en Educación Superior un estudio de egresados, Revista electrónica Actualidades Investigativas en Educación, ISSN: 1409-4703 (en línea), 6(3), (2006). http://redalyc.uaemex.mx/src/inicio/ArtPdfRed.jsp?iCve=44760306\#, Acceso el 1 de junio (2011).

Fernández, M y Rodríguez, N. Seguimiento de egresados: obstáculos y beneficios. Revista Omnia. ISSN: 1351-8856, 5(2), (1986).

http://www.posgrado.unam.mx/servicios/productos/omnia/anteriores/33/11.pdf. Acceso: 17 de Noviembre (2011).

Fernández, Ma. (1992) Primicias del impacto de los egresados de la Maestría en Administración de la UPIICSA en las empresas. Revista Omnia, ISSN: 1351-8856, (8)(359-364), Número especial. 
Fernández, Ma. (1993) Calidad del desempeño del egresado de la maestría en Administració de la UPIICSA: opinión de los ejecutivos. Revista Omnia, ISSN: 1351-8856, 9(253-257). Número especial.

Figueroa, S., Bernal, B. y Andrade, C.K. Evaluación de un programa mexicano de maestría en psicología desde la perspectiva del egresado: un estudio sobre los indicadores de calidad. Revista de Educación Superior, ISSN 0185-2760 (en línea), 39(153), Enero-Marzo (2010), http://www.scielo.org.mx/scielo.php?pid=S0185-27602010000100002\&script=sci_abstract. Acceso: el 15 de noviembre (2011).

Fox, D. y López, E. El proceso de investigación en educación, 1ª edición, (367-369), Universidad De Navarra, España (1981).

Fresán, M. Los estudios de egresados. Una estrategia para el autoconocimiento y la mejora de las Instituciones de Educación Superior, en Esquema Básico para el estudio de egresados, $1^{\mathrm{a}}$ edición, Colección Bibliotecas de la Educación Superior ANUIES, (19-31), México, (1998).

Hernández, R., Fernández, C. y Baptista, P. Metodología de la investigación, 4ta edición, (160209), McGraw Hill, México (2006).

Luchilo, L. Los impactos del programa de becas del CONACYT mexicano: un análisis sobre la trayectoria ocupacional de los ex becarios (1997-2006). Revista iberoamericana de ciencia tecnología y sociedad, ISSN: 1850-0013 (en línea), 5(12), (2009).

http://www.scielo.org.ar/scielo.php?pid=S1850-00132009000200010\&script=sci_abstract. Acceso: 20 de noviembre (2011).

Manzo, L., Rivera C. y Rodríguez, A. R. La educación de posgrado y su repercusión en la formación del profesional iberoamericano. Revista Educación Médica Superior, ISSN 0864-2141 (en línea), 20(3), julio-septiembre (2006),

http://scielo.sld.cu/scielo.php?script=sci_arttext\&pid=S0864-

$21412006000300009 \&$ Ing=es\&nrm=iso. Acceso 13 de Noviembre del 2011.

Martínez, G., Moreno, B., Hernández, C., Miguel, G., y Martínez, F. Los egresados del posgrado de la UNAM, Revista de Educación Superior, ISSN: 0185-2760 (en línea), 34(133), enero-marzo (2005). http://redalyc.uaemex.mx/redalyc/src/inicio/ArtPdfRed.jsp?iCve=60411915003, Acceso: el 10 de junio de (2011).

Ramírez, C., Reyna, M., García, A., Ortiz, X y Valdez, P. Formación científica de los egresados de tres programas de maestría en ciencias: seguimiento a 10 años (1999-2009). Revista de la Educación Superior, ISSN 0185-2760, 2(158), Abril-Junio. Asociación Nacional de Universidades e Instituciones de Educación Superior (2011).

Sánchez, R. y Arredondo, M. Pensar el posgrado. La eficiencia terminal en ciencias sociales y humanidades de la UNAM, $1^{\text {a }}$ edición, 117-122, Universidad Nacional Autónoma de México, México (2001).

Secretaría de Educación Pública. Sistema educativo de los Estados Unidos Mexicanos, principales cifras, ciclo escolar 2008-2009, 1ª edición, 164-170, Dirección General de Planeación y Programación, México (2009).

Valenti, G. y Varela, G. Diagnóstico sobre el estado actual de los estudios de egresados, $1^{\mathrm{a}}$ edición, 9-20, Colección documentos, Asociación Nacional de Universidades e Instituciones de Educación Superior, México (2004). 
